\title{
Advances in Clinical Decision Support: Highlights of Practice and the Literature 2015-2016
}

\author{
R. A. Jenders ${ }^{1,2}$ \\ 1 Center for Biomedical Informatics and Department of Medicine, Charles Drew University, Los \\ Angeles, California, USA \\ 2 Clinical and Translational Science Institute and Department of Medicine, University of California, \\ Los Angeles, California, USA
}

\section{Introduction}

Clinical decision support (CDS) has been defined as a process for enhancing health-related decisions and actions with pertinent, organized clinical knowledge, and patient information to improve health and health care delivery [1]. This broad process has been characterized as improving outcomes by addressing the five "rights": delivering the right, evidence-based information to the right people, including clinicians and patients, in the right format through the right channels at right times [2]. Indeed, CDS is not simply an alert, reminder, or explicit care suggestion, but instead it encompasses a variety of tools, including alerts and reminders for patients and physicians, clinical guidelines, order sets, focused patient data reports, documentation templates, diagnostic support, and contextually relevant reference information [3]. Much of the activity in this area is driven by market and regulatory contexts with a resulting emphasis on using health information technology for quality improvement. This has included the previously implemented regulatory framework and incentive scheme of meaningful use in the USA. More specifically in this context, CDS has been defined as health information technology functionality that builds upon the foundation of an electronic health record system to provide persons involved in care processes with general and person-specific information, intelligently filtered and organized, at appropriate times, to enhance health and health care [4]. Clinical decision support has been shown to improve the process of care by improving health care provider performance, although its demonstrated effect on clinical outcomes has been much more limited, albeit in part because of methodological limitations in studies of CDS [5].

With this very broad context, CDS continued to play a significant and expanding role in health care, health science, and the practice of clinical informatics during the period 2015-2016. No single unifying theme unites this body of work, but a number of diverse themes run through it. In addition to quality improvement, researchers continue to address the challenges of big data in an era of precision medicine, including the provision of tools to help patients and clinicians make good decisions using large amounts of possibly unfamiliar data [6]. Continued expansion of this technology to different clinical contexts, such as emergency medicine, is another hallmark of activity in CDS [7]. Despite efforts over many years, researchers also continue to seek ways to implement clinical practice guidelines using health information technology, including expanding use of mobile technology [8].

In light of these myriad factors, motivations and incentives, and following the tradition of the IMIA Yearbook, the principal objective of the present article is to provide a survey of the most recent health science literature and the practice of clinical informatics with regard to CDS. Considering the past two years, 2015-2016, it aims at highlighting key themes in the field of CDS. This includes scientific research, 
clinical practice, and development of health information technology (HIT) standards to facilitate this activity. Ultimately, these diverse efforts will expand the boundaries of this technology in ways that will improve research, clinical practice, and health outcomes.

\section{Methods}

Key sources of evidence related to CDS activity were reviewed, with a focus on the period 2015-2016. These included the proceedings of the World Congress of Medical Informatics (Medinfo 2015) and those of the annual symposium of American Medical Informatics Association (AMIA 2016) as premier forums for the presentation of contemporary, cutting edge work. MEDLINE references with a date of publication in the 2015-2016 time frame matching a text word search of "clinical decision support" were also reviewed with a focus on a publication type of review. In addition, standardization activities of Health Level Seven International (HL7), the premier standards development organization in the HIT space, were reviewed as a representation of the role HIT standards can play in CDS. Consistent with a grounded theory approach to the analysis of qualitative, narrative data [9], key codes or concepts in the text of publications from these sources that described the activity of CDS or its outcome were identified. These concepts then were grouped based on semantic similarity into broad categories in order to generate the CDS themes presented here. For example, different papers could contain concepts such as "mobile sensor data use", "bedside instrumentation", and "error reduction", and these could be grouped in thematic analysis into the theme "precision medicine". This approach facilitated aggregation of diverse subjects into key themes that help to identify the state of the art and science of CDS in recent years and to help forecast the direction of the field in the near future. To concretize and elaborate on each of these themes, a selected, small number of illustrations from the literature and practice are provided.

\section{Results}

Like any aspect of health care delivery, research and practice, the development and use of CDS respond to its environment. In particular, this includes regulatory regimes established by governments, financial incentives, and the standards of professional societies and other stakeholder organizations. These influences, such as the US Medicare and Children's Health Insurance Program Authorization Act of 2015 (MACRA) and its effect on efforts related to the drive for meaningful use of electronic health record systems in the USA and for quality improvement [10], can shape the use of CDS through the use of financial and regulatory incentives. Other regulatory efforts, both in North America and the European Union, focusing on decision support software, including that incorporated in or classified as medical devices, can stimulate or otherwise may affect work in CDS [11]. Further, directed research funding, such as that provided by the European Union for the Platform for European Preparedness
Against Re-emerging Epidemics (PREPARE) that helps to promote a learning health system that merges clinical trials and decision support into a continuous learning process, also can shape the practice of CDS [12].

These environmental factors in turn affect the practice of CDS and the body of research published about CDS. Taking these effects into account, workers have defined models that delineate the key types of CDS systems and interventions that have emerged as a response [13, 14]. Rather than duplicate these models, the present survey of recent works in CDS identifies ten thematic areas that involve CDS systems or fortify the infrastructure necessary for them to be used to peak potential (Table 1). Certainly these thematic areas can be aggregated in a variety of ways, and individual projects may pertain to more than one of these thematic areas. Nevertheless, the large diversity of work in CDS in the recent past confirms it as an important domain for research and practice.

Table 1 Summary of the 10 key thematic areas of clinical decision support (CDS) work during the period 2015-2016 (HIT: health information technology; (POE: computer-based provider order entry).

\begin{tabular}{|c|c|}
\hline Theme & Summary \\
\hline Knowledge Representation & $\begin{array}{l}\text { Structuring knowledge for processing by computer-based } \\
\text { decision support systems }\end{array}$ \\
\hline Precision Medicine & $\begin{array}{l}\text { Using large data sets, including genomic and sensor data, to } \\
\text { provide highly tailored CDS }\end{array}$ \\
\hline Quality Improvement & Enhancing the safety and outcomes of clinical care \\
\hline CPOE Systems & $\begin{array}{l}\text { Providing CDS at the time that clinicians write orders } \\
\text { regarding diagnostic evaluation or treatment }\end{array}$ \\
\hline Expansion of Clinical Settings & $\begin{array}{l}\text { Using CDS in care settings such as the emergency department } \\
\text { and in domains less emphasized in the past such as } \\
\text { anesthesiology and nursing }\end{array}$ \\
\hline Patient-Directed CDS & $\begin{array}{l}\text { Exposing patients to CDS instead of, or in addition to, the } \\
\text { more traditional clinician target }\end{array}$ \\
\hline Negative Impact of CDS & $\begin{array}{l}\text { Identifying ways that CDS can adversely affect clinical care } \\
\text { and outcomes }\end{array}$ \\
\hline Structuring Data for CDS & $\begin{array}{l}\text { Coding data and employing controlled terminologies to make } \\
\text { data more usable in CDS }\end{array}$ \\
\hline Diagnostic Decision Support & Using CDS to improve the accuracy of diagnosis \\
\hline HIT Standards for CDS & $\begin{array}{l}\text { Providing standard interfaces, data models, and inference } \\
\text { mechanisms to ease the implementation of CDS }\end{array}$ \\
\hline
\end{tabular}




\section{Knowledge Representation}

Much activity in CDS continues to center on the exploration of ways to use the knowledge compiled in clinical practice guidelines in order to enhance adherence to them and thereby improve the quality of the process and outcomes of health care. This includes efforts to structure guidelines and improve their computability, as well as more generally how to manage scientific and expert knowledge for use by clinicians and patients.

For example, the treatment of asthma continues to be a clinical domain where clinical practice does not meet evidence-based guidelines. The ARIA international effort has led to the use of CDS technology to improve care of patients with asthma or rhinitis [8]. The prevention of venous thromboembolism remains a vexing clinical challenge, yet multidisciplinary efforts that leverage CDS, including synchronous alerting and order sets, have made progress in this area [15]. Some CDS efforts with guidelines have involved their use in somewhat non-traditional settings, such as educational efforts that have improved process outcomes in pharmacy practice [16]. A key part of implementing computable guidelines concerns identifying where, at any given moment, a particular patient is on the clinical pathway endorsed by a guideline, and recent work in modeling clinical trajectories has improved our knowledge in this regard [17]. A key aspect of knowledge management is reusing computable knowledge, and work at Vanderbilt University, inter alia, is helping to elaborate ways to curate and reuse knowledge in CDS systems [18]. Even independent of the use of HIT, checklists have emerged as powerful instruments in health care and other industries, such as aviation, and recent work in CDS that facilitates the use of checklists to improve performance in critical care situations highlights this trend [19].

While the use of guidelines as part of knowledge representation has been important in recent work, other approaches have been employed. Work that combines different formalisms, such as CDS that can improve the ability of practitioners to diagnose cancer by combining case-based reasoning with a rule-based approach, offers promise [20]. Ontology-based CDS, such as a system to help manage patients with numerous chronic problems that potentially can involve multiple guidelines, continues to be used [21]. In addition, automated identification of temporal aspects of care pathways can help improve their use [22]. Implementation of guidelines in a way that uses dynamic adjustment according to a patient's own laboratory data in order to detect potentially dangerous states addressed by guidelines may enhance patient safety [23]. Knowledge-based interventions using novel approaches that leverage other areas, such as cross-fertilization involving more traditional audit and feedback, holds promise [24]. Because competing guidelines addressing the same clinical topic can produce different recommendations, work that addresses the synthesis of such CDS output, including a framework for the fusion of treatment recommendations, is important [25]. Finally, recognizing that while CDS may improve guideline adherence, it is not a panacea, and work to understand the reasons why clinicians do not accept CDS-generated recommendations, such as those regarding the management of breast cancer patients, is important [26].

\section{Precision Medicine}

Analytics involving the use of "big data", such as genomic and proteomic data as well as ambulatory and related sensor data, in conjunction with CDS in order to try to personalize clinical care and thereby improve its effectiveness, was a key area of recent work in CDS. The use of large collections of clinical and laboratory data for forecasting that can feed into CDS efforts shows promise, such as predicting treatment responses in radiation oncology [27]. The establishment of learning health systems, which are structured in part to acquire knowledge from clinical practice and experimentation that can be fed back into CDS efforts to improve care, is a growing phenomenon, and the resulting massive data sets that emerge from such collaboration can be used to improve care in areas such as pediatric surgery [28]. The use of sensor data and mobile technology to support CDS interventions, including self-management thereof, may provide value in a world of seemingly ubiquitous mobile devices [29]. Integration of bedside instrumentation with a CDS system has been shown to reduce charting time, increase time spent on direct care, reduce errors, and improve clinical outcomes [30].

In addition, use of particular techniques to increase the utility of big data has shown interesting results, such as the employment of an artificial neural network and deep-learning techniques to predict inpatient mortality and potential benefit from clinical interventions [31]. Identification of high risk for complications from heart failure using large data sets also offers a pathway for CDS that can improve outcomes [32]. Using pharmacogenomic data to drive CDS in ways that can personalize care, such as efforts in the eMERGE Network, highlights the important interaction between precision medicine and CDS [33]. Leveraging big data to explore interactions among clinical and genetic factors using Bayesian networks to classify manifestations of inflammatory bowel disease illustrates other possibilities of CDS in the realm of precision medicine [34]. Leveraging the promise of the Internet of Things for acquiring data and providing information through the design of a reference architecture that can be used to implement CDS illustrates the potential in this area [35].

\section{Quality Improvement}

A key promise of CDS is the potential for improving the process of clinical care and its health outcomes. This flows in part from work designed to detect abnormal patient states or events and to communicate the need for action to decision-makers. For example, work to detect and even predict adverse drug reactions can help to improve quality by reducing the negative consequences of clinical care [36]. Automated techniques for knowledge discovery that can inform CDS work to improve medication safety also emphasize quality improvement [37]. Additional work in this area shows how the use of patient data coupled with CDS that detects early clinical deterioration in, for example, the pediatric inpatient setting, including the use of patient dashboards to inform treatment that intervenes before serious deterioration, may improve the quality of care [38]. 


\section{Computer-based Provider Order Entry}

Computer-based provider order entry (CPOE) is a key technology that offers considerable promise to influence decisions, change practice, and improve clinical care, through the integration of CDS, patient data, and clinical care management. By bringing together the clinician, relevant patient data, and other forms of CDS at the time that decisions are being made about care in a computer-based environment, as substantiated by recent work, CPOE systems can improve care. For example, even if empiric validation may be limited, the use of CPOE technology in a pediatric electronic health record system may be valuable for care quality [39]. In an example that flows from recent regulatory changes in the USA to emphasize guideline-appropriate ordering of imaging studies, work has demonstrated that such technology can increase appropriate use of imaging studies by a moderate amount and decrease inappropriate use by a small amount [40]. Additional evidence suggests that the use of this technology can exert a moderate effect on improving antibiotic prescribing in the ambulatory environment [41].

On the other hand, evidence has arisen suggesting that the commercial market for CPOE systems, such as that in England, is at an immature stage of development that does not meet user needs even as it is further hampered by unrealistic adopter demands [42]. These observations were derived from semi-structured interviews with and ethnographic observations of system users, and they substantiated complaints that overly generic configurations are not sufficiently specialized for local user needs. However, these data also revealed that users may not be aware of the characteristics and limitations of packaged systems and that this lack of awareness may lead to unrealistic expectations that in turn could lead to system dissatisfaction [42]. Moreover, even systems that are certified under regulatory schemes may lack the capability to implement certain kinds of decision support rules [43]. Indeed, there is increasing recognition that $\mathrm{CPOE}$ and related technology can malfunction in ways that cause problems, which poses at least a potential threat to patient safety
[44]. Nevertheless, there is recognition that an emphasis on user interface design issues may hold the key to improving the utility of this technology, such as simulation work with potential users that can help identify potential failure points [45].

\section{Expansion of Clinical Settings}

In the recent past, workers continued to expand the domains, settings, and targets of CDS in ways that illustrate its value, including different clinical disciplines and the use of CDS in clinical research. For example, as a specialized environment characterized by high volume of cases, a wide range of clinical presentations, significant time-pressure, and high severity, the emergency department particularly could benefit from CDS. A recent review documents that approximately half of 23 selected studies demonstrated a positive effect of CDS on care in this setting [7]. CDS has demonstrated benefits for patient care in anesthesiology via point-of-care technology, particularly in ordering antibiotic prophylaxis, and in the use of clinical documentation [46]. While the origins of CDS can be traced back to physician-directed support, more recent work has highlighted its impact among other groups of clinicians. Indeed, in a recent review examining the impact of CDS on nurses, all the studies that included process outcomes and usability outcomes, and also had analytic procedures to detect changes in outcomes, demonstrated statistically significant improvements [47].

In another work focusing on different domains, recent evidence has shown that, despite sometimes ineffective outcomes that can result in part from low use of CDS functionality, use of CDS to provide diabetes care can be improved by prioritizing care recommendations, improving communication of treatment-relevant information to patients, using such systems for care coordination and case management, and integrating patient-reported information and data from remote devices [48]. In other settings, CDS continues to hold promise for improving public health through disease surveillance and helping to intervene against outbreaks, including recent work to model spread of the Zika virus in dynamic human and vector populations [49]. Expansion of the use of CDS in psychiatry has produced interesting results that appear to improve on current clinical practice on predicting the development of post-traumatic stress disorder after traumatic events [50] and in predicting suicide [51]. Applying CDS in the realm of clinical research requires not just appropriate data access but also methods for processing and interpreting research protocols and informed consent documentation. Work in natural language processing identifies ways to structure such knowledge for potential use in CDS [52]. Work in matching patients to potential clinical trials remains challenging; recent efforts involving a semantic web approach yielded high recall but relatively low precision, mainly due to missing data in the patient record [53]. Nevertheless, despite all this potential in these expanded domains of CDS application, the effect of CDS on patient-reported outcomes may be only marginally positive [54].

\section{Patient-Directed Clinical Decision Support}

While traditionally intended to help improve decision-making by clinicians caring for patients, CDS has been increasingly developed for patients themselves as the individuals with the most at stake in any given health care decision and as the final common pathway for effecting many treatments, such as medication administration and lifestyle modifications. For example, workers are identifying uses of mobile technology to help train adolescents, who comprise a disproportionate share of those at risk for anaphylaxis, about the appropriate use of epinephrine auto-injection [55].

Initiatives that target patients for decision support use methods taking into account factors such as suitability for diverse patient populations, including work to develop a patient-directed decision aid for those with heart failure who may need a left ventricular assist device, which effort required 19 iterations to produce suitable written and video decision support material for patients [56]. Indeed, in additional evidence pertaining to 
educating patients about oncologic disease, researchers who developed computer-based decision support for patients with thyroid cancer discovered only a moderately positive correlation of knowledge acquisition with CDS use [57], suggesting the need for additional work in this important thematic area. Future work to fold shared-decision making between clinicians and patients into CDS, such as implementation of the direct decision support model being promoted by the US Centers for Medicare and Medicaid Services (CMS), likely will help address these challenges [58].

\section{Negative Impact of Clinical Decision Support}

In addition to the many benefits of HIT, there is growing recognition that use of HIT in general and of CDS in particular could result in negative impacts on patients, including direct harm. For example, in a recent review that examined the causes of medication prescribing errors in the use of CPOE systems [59], workers identified CDS as one of the causes of such errors. In addition, the literature in this thematic area has described the challenge of alert fatigue with CPOE and other systems that use synchronous alerts to implement CDS. One aspect of this are the high override rates for some alerts in some systems, such as an override rate as high as $89 \%$ for opioid drug-allergy alerts [60]. This has progressed to the point where workers more recently have developed efforts to address this problem. For example, researchers at Dalhousie University in Nova Scotia have created the INITIATE framework to enable intelligent adaption to the output of alert-generating systems in a way that could help alleviate alert fatigue [61]. Additional work to reduce alert fatigue related to drug-drug interaction alerts, including targeted alert reduction as measured by "think time"-a metric for evaluating the resolution time for an alert-managed to reduce the alert burden overall as well as improve prescriber efficiency [62]. Accordingly, while evidence demonstrates that HIT and CDS can have a negative impact, recent work also has shown pathways for addressing such an impact.

\section{Structuring Data}

Computer-based CDS essentially relies on the application of knowledge to data in digital format in order to improve decision-making. While sometimes not directly related to CDS, attempts to improve the availability of structured data, including representation using data models and controlled terminologies, can be vital in the accuracy and ultimate success of CDS. For example, workers at Partners Healthcare in Boston have shown how their strong efforts over a decade to derive structured data from clinical documentation can serve as the building blocks for leveraging data to improve care, including via CDS [63]. Other work has shown how training techniques can be used to improve the performance of natural language parsers with regard to semantic role labeling, which can help improve the structure of narrative data for use in activities such as CDS [64]. Structuring complex data such as microbiology reports can help improve their utility for secondary uses, including CDS [65]. The use of a reference terminology in this context, such as SNOMED CT, can help evaluate terminology coverage in a CDS system and possibly improve its recognition of data in a patient's electronic health record [66]. In large health care organizations, a systematic approach, such as the ten-step process employed at Partners Healthcare, can assist greatly in the provision of structured data for CDS and other purposes [67].

\section{Diagnostic Clinical Decision Support}

As an important case of secondary use for structured clinical data, diagnostic decision support dates to the earliest days of the academic endeavor that later became biomedical informatics. Recent work continues to emphasize the importance of assisting clinicians and patients in the early and accurate diagnosis of disease so that curative interventions can be undertaken. For example, at least observational evidence suggests that expedited diagnosis of symptomatic cancer may provide benefits, and initiatives to accomplish this in the primary care setting similarly may be beneficial [68]. Beyond structured clinical data, approaches for improving diagnosis using image data, such as photographs of the ocular fundus, may result in better care [69]. In another effort to prevent harm by improved diagnostic detection, the use of a multi-algorithm approach to detecting patient-ventilator asynchronies that can result in lung injury can improve detection in ventilator wave form data from $66 \%$ to as high as $95 \%$ [70]. A variety of score calculators to identify risks for and probability of presence of various diseases already exist, but a key challenge remains to integrate them with electronic health record systems. Recent work has demonstrated that, even with advanced techniques for variable extraction, only $29 \%$ of 171 such scores are completely programmable, while $63 \%$ are at least $75 \%$ programmable, suggesting that such integration holds both promises and challenges [71].

\section{Health Information Technology Standards for Clinical Decision Support}

Research has demonstrated that HIT standards have a positive role to play to get the structured data needed for CDS, such as facilitating data exchange in clinical research using the CDISC Operational Data Model (ODM) to support exchange of research protocols and case reports [72]. In addition, HIT standards play a role in the structuring of and access to knowledge that can form the basis of CDS interventions. Indeed, the presence of numerous standards in this space may interfere with the semantic interoperability needed to share computable knowledge among CDS systems [73]. In addressing these challenges, some workers have focused on improving standardization of data representation within CDS knowledge formalisms, such as the potential use of the HL7 standard Fast Healthcare Interoperability Resources (FHIR) as a possible standard data model in the Arden Syntax [74].

Sidestepping the challenge of agreeing a standard formalism that allows computable knowledge to be shared, some workers have emphasized standards that allow the integration of CDS applications with electronic health record systems, such as the increasingly popular CDS Hooks protocol [75]. 
Other workers have incorporated support for sophisticated reasoning techniques in CDS knowledge formalisms, such as the incorporation of fuzzy logic in the Arden Syntax in order to mimic clinical reasoning regarding infection surveillance [76]. Additional work in standards has demonstrated the significant prevalence of fuzzy logic in clinical reasoning and shown how a standard such as the Arden Syntax that incorporates constructs for fuzzy logic can be used to represent the knowledge in a large corpus of quality measures [77]. Widespread agreement on a standard CDS knowledge formalism such as the Arden Syntax remains elusive even if this approach has demonstrated utility in implementing CDS [78]. Standards development organizations such as HL7 seek to address the utility of such standards not only for knowledge sharing but also for knowledge access, such as improvements in the Infobutton standard, a popular method for accessing knowledge, e.g. genomic resources, within electronic health record systems [79].

\section{Discussion}

Overall, the current work is a thematic, selective survey of recent activity in CDS covering a recent time period that aligns with the timeline and requirements of the IMIA Yearbook. It is a characterization of the kinds of recent activities in CDS, particularly research, in order to provide an overview of this field. Differing from more elaborate recent studies [80], it is not a top-down, comprehensive systematic review addressing all possible facets of CDS. Instead, this survey elaborates key themes in research and practice involving CDS during this time period derived from the narrative text of relevant publications. Moreover, while illustrative examples of these themes are described, space does not allow inclusion of every single study or effort relevant to the identified themes. The overall objective was to convey the breadth of activity in the important domain of CDS. As manifested by the illustrative examples in the ten identified thematic areas, our knowledge of appropriate structure, implementation, utility, and effective CDS continues to grow. Indeed, the illustrated effects, as well as actual and potential utility of CDS, offer potentially pervasive effects on clinical practice and health care delivery, making a review of recent work all the more timely.

Acting on this imperative, other workers have conducted reviews of research and practice in CDS but elaborated somewhat different classifications. For example, in their comprehensive quarter-century review of CDS published in 2016, Middleton and colleagues identified a framework through iterative discussion among themselves that framed their informal review of CDS around six axes: data, knowledge, inference, architecture and technology, implementation and integration, and users [80]. A decade earlier, Peleg and $\mathrm{Tu}$, focusing in part on knowledge representation and management in the context of CDS, framed their analysis of CDS using a life-cycle approach [81]. Their thematic areas included the goals of CDS, CDS systems as part of a knowledge management enterprise, modeling of knowledge to enable knowledge representation, design features that foster success of CDS systems, standardization efforts, and evaluation of CDS.

The present analysis, which covers a more limited time frame than the abovementioned studies, differs in methodology and offers both contrasts with and similarities to these prior classifications. All three analyses identify an emphasis on knowledge structure and representation in CDS in research and practice in this area. Further, while aggregating the themes somewhat differently, all three cover the importance of standards and evaluation, although the present analysis does this in part through the theme of the negative impact of CDS. By contrast, because it is somewhat more granular than the earlier studies, the present elaboration of CDS themes suggests the emphasis on specific uses of CDS, such as quality improvement and the use of CDS to help practice precision medicine.

One point brought forth by comparing different classifications of CDS activity is that overlap may occur between classification schemes and within any particular example used to illustrate them. For example, a single study may use a standard knowledge formalism to provide the computable knowledge that then, implemented in a CDS system, is used to improve clinical outcomes. Such a study could be classified in at least three themes identified in the present classification: knowledge representation, quality improvement, and HIT standards. Nevertheless, the key purpose in the present analysis was to identify key kinds of CDS activity even if every single published work could not be discretely classified into a single theme.

Despite the progress illustrated by the examples of each of the CDS themes, barriers and limitations continue to affect the practice of CDS. Financial incentives may not be fully aligned to promote quality over volume in a way that could increase uptake of CDS; despite considerable efforts to contrary, lack of widespread use of knowledge representation and terminology standards makes knowledge sharing and data use in CDS difficult; and re-engineering is not infrequently needed to implement knowledge bases at different sites [80]. Moreover, despite recommendations to the contrary, adequate governance, knowledge maintenance, and feedback from evaluation may not be in place to make ideal use of CDS [1].

Regardless of the varying ways CDS is being implemented and the barriers to or limitations on its use, the application of knowledge remains central, and the recent work highlighted here addresses both parts of this milieu. Knowledge is addressed through the development of HIT standards for representing, sharing, and accessing it; by leveraging clinical practice guidelines to incorporate evidence-based practice recommendations in CDS; by improvements in support for diagnostic activity; and by the expansion into different clinical domains and applications, such as clinical research and nursing. The role of data in CDS is addressed by increasing big data to facilitate precision medicine and by structuring data, including the use of standard data models and terminologies. The overall utility of CDS has been demonstrated by work in quality improvement and by research in patient-directed decision support. While much of this work shows promises for achieving the benefits attributed to CDS, including improvements in the process and, to a more limited extent, clinical outcomes of health care, recent work also demonstrates that CDS is not a panacea and that care must be taken to minimize its adverse consequences as well. 


\section{Conclusions}

Recent work in CDS has demonstrated both potential and pitfalls in improving the process and outcomes of clinical care. While several models have been identified for classifying CDS and CDS systems, recent work in CDS can be aggregated in ten thematic areas. The breadth and depth of research and practice in CDS in recent years substantiate its importance and offer promise for still more advances in the future.

\section{Acknowledgements}

This work was supported in part by NIMHD grants U54MD007598 and S21MD000103 and NCATS grant UL1TR001881 from the National Institutes of Health (USA).

\section{References}

1. Osheroff JA, Teich JM, Levick D, Saldana L, Velasco F, Sittig D, et al. Improving Outcomes with Clinical Decision Support: An Implementer's Guide, 2 ed. Chicago: Healthcare Information and Management Systems Society; 2012.

2. Sirajuddin AM, Osheroff JA, Sittig DF, Velasco F, Collins DA. Implementation pearls from a new guidebook on improving medication use and outcomes with clinical decision support. Effective CDS is essential for addressing healthcare performance improvement imperatives. J Healthc Inf Manag 2009;23(4):38-45.

3. Center for Medicare and Medicaid Services. Clinical decision support: more than just 'alerts' tipsheet. https://www.cms.gov/Regulations-and-Guidance/ Legislation/EHRIncentivePrograms/Downloads/ ClinicalDecisionSupport_Tipsheet-.pdf. Accessed 1 November 2016.

4. Medicare and Medicaid Programs - Electronic Health Record Incentive Program. Final rule. Fed Regist 2010 Jul 28;75(144):44314-44588.

5. Jaspers MW, Smeulers M, Vermeulen H, Peute LW. Effects of clinical decision support systems on practitioner performance and patient outcomes: a synthesis of high-quality systematic review findings. J Am Med Inform Assoc 2011;18(3):327-34.

6. Korngiebel DM, Thummel KE, Burke W. Implementing precision medicine: the ethical challenges. Trends Pharmacol Sci 2017;38(1):8-14.

7. Bennett P, Hardiker NR. The use of computerized clinical decision support in emergency care: a substantive review of the literature. J Am Med Inform Assoc 2017 May 1;24(3):655-68.

8. Bousquet J, Hellings PW, Agache I, Bedbrook A, Bachert C, Bergmann KC, et al. ARIA 2016: Care pathways implementing emerging technologies for predictive medicine in rhinitis and asthma across the life cycle. Clin Transl Allergy 2016;6:47.

9. Chapman AL, Hadfield M, Chapman CJ. Qual- itative research in healthcare: an introduction to grounded theory using thematic analysis. J R Coll Physicians Edinb 2015;45:201-5.

10. Bekelis K, McGirt MJ, Parker SL. The present and future of quality measures and public reporting in neurosurgery. Neurosurg Focus. 2015 Dec;39(6):E3.

11. Yang YT, Thompson BM. Regulatory framework for clinical decision support software: present uncertainty and prospective proposition. J Am Coll Radiol 2015;12(7):672-5.

12. Lewis RJ. The pragmatic clinical trial in a learning health care system. Clin Trials 2016;13(5):484-92.

13. Sim I, Berlin A. A framework for classifying decision support systems. AMIA Annu Symp Proc 2003;2003: 599-603.

14. Wright A, Sittig DF, Ash JS, Feblowitz J, Meltzer S, McMullen C, et al. Development and evaluation of a comprehensive clinical decision support taxonomy: comparison of front-end tools in commercial and internally developed electronic health record systems. J Am Med Inform Assoc. 2011 May-Jun;18(3):232-242.

15. Streiff MB, Lau BD, Hobson DB, Kraus PS, Shermock KM, Shaffer DL, et al. The Johns Hopkins Venous Thromboembolism Collaborative: multidisciplinary team approach to achieve perfect prophylaxis. J Hosp Med 2016;11 Suppl 2:S8-S14.

16. Watkins K, Wood H, Schneider CR, Clifford R. Effectiveness of implementation strategies for clinical guidelines to community pharmacy: a systematic review. Implement Sci. 2015 Oct 29;10:151.

17. Dabek FJ, Caban JJ. A k-reversible approach to model clinical trajectories. AMIA Annu Symp Proc 2016;2016:460-9.

18. DesAutels SJ, Fox ZE, Giuse DA et al. Using best practices to extract, organize, and reuse embedded decision support content knowledge rules from mature clinical systems. AMIA Annu Symp Proc 2017 Feb 10;2016:504-13.

19. Sarcevic A, Zhang Z, Marsic I, Burd RS. Checklist as a memory externalization tool during a critical care process. AMIA Annu Symp Proc 2016;2016:1080-9

20. Saraiva RM, Bezerra J, Perkusich M, Almeida $\mathrm{H}$, Siebra C. A hybrid approach using case-based reasoning and rule-based reasoning to support cancer diagnosis: a pilot study. Stud Health Technol Inform 2015;216:862-6.

21. Galopin A, Bouaud J, Pereira S, Seroussi B. An ontology-based clinical decision support system for the management of patients with multiple chronic disorders. Stud Health Technol Inform 2015;216:275-9.

22. Li X, Liu H, Mei J, Yu Y, Xie G. Mining temporal and data constraints associated with outcomes for care pathways. Stud Health Technol Inform 2015;216:711-5.

23. Fraccaro P, Brown B, Prosperi M. Development and preliminary validation of a dynamic, patient-tailored method to detect abnormal laboratory test results. Stud Health Technol Inform 2015;216:701-5.

24. Brown B, Peek N, Buchan I. The case for conceptual and computable cross-fertilization between audit and feedback and clinical decision support. Stud Health Technol Inform 2015;216:419-23.

25. Mei J, Liu H, Li X, Xie G, Yu Y. A decision fusion framework for treatment recommendation systems. Stud Health Technol Inform 2015;216:300-4.

26. Bouaud J, Spano JP, Lefranc JP, Cojean-Zelek I, Blaszka-Jaulerry B, Zelek L, et al. Physicians' attitudes towards the advice of a guideline-based decision support system: a case study with OncoDoc2 in the management of breast cancer patients. Stud Health Technol Inform 2015;216:264-9.

27. Lambin P, Zindler J, Vanneste BG, De Voorde LV, Eekers D, Compter I. Decision support systems for personalized and participative radiation oncology. Adv Drug Deliv Rev 2017 Jan 15;109:131-53.

28. Hsiung GE, Abdullah F. Improving surgical care for children through multicenter registries and QI collaboratives. Semin Pediatr Surg 2015 Dec;24(6):295-306.

29. Steinhubl SR, Muse ED, Topol EJ. The emerging field of mobile health. Sci Transl Med 2015 Apr 15:7(283):283rv3.

30. Cheung A, van Velden FH, Lagerburg V, Minderman N. The organizational and clinical impact of integrating bedside equipment to an information system: a systematic literature review of patient data management systems (PDMS). Int J Med Inform 2015 Mar;84(3):155-65.

31. Aczon M, Ledbetter D, Van Ho L, Gunny A, Wetzel R. Deep learning recommendation of treatment from electronic data. AMIA Annu Symp Proc 2016;2016:5-6

32. Evans RS, Benuzillo J, Horne BD, Lloyd JF, Bradshaw $\mathrm{A}$, Budge $\mathrm{D}$, et al. Automated identification and predictive tools to help identify high-risk heart failure patients. AMIA Annu Symp Proc 2016;2016:53-54

33. Herr TM, Peterson JF, Rasmussen LV, Caraballo PJ, The eMERGE Network. Implementing pharmacogenomic clinical decision support: design and prescriber response in the eMERGE Network. AMIA Annu Symp Proc 2016;2016:61-62.

34. Menti E, Lanera C, Lorenzoni G, Giachino DF, Marchi M, Gregori D, et al. Bayesian machine learning techniques for revealing complex interactions among genetic and clinical factors in association with extra-intestinal manifestations in IBD patients. AMIA Annu Symp Proc 2016;2016:884-93.

35. Gerdes M, Reichert F, Nytun JP, Fensli R. Future telehealth and telecare reference design based on iot technologies: from remote monitoring to smart collaborative services with decision support. Stud Health Technol Inform 2015;216:891.

36. Kane-Gill SL, Achanta A, Kellum JA, Handler SM Clinical decision support for drug related events: Moving towards better prevention. World J Crit Care Med 2016;5(4):204-11.

37. Tan Y, Hu Y, Liu X. Improving drug safety: From adverse drug reaction knowledge discovery to clinical implementation. Methods 2016 Nov 1;110:14-25.

38. Suresh S. Big data and predictive analytics: applications in the care of children. Pediatr Clin North Am 2016 Apr;63(2):357-66.

39. Dufendach KR, Eichenberger JA, McPheeters ML, Temple MW, Bhatia HL, Alrifai MW, et al. Core functionality in pediatric electronic health records. Rockville (MD): Agency for Healthcare 
Research and Quality (US); 2015 Apr. Report No.: 15-EHC014-EF

40. Goldzweig CL, Orshansky G, Paige NM, Miake-Lye IM, Beroes JM, Ewing BA, et al. Electronic health record-based interventions for improving appropriate diagnostic imaging: a systematic review and meta-analysis. Ann Intern Med 2015 Apr 21;162(8):557-65.

41. Holstiege J, Mathes T, Pieper D. Effects of computer-aided clinical decision support systems in improving antibiotic prescribing by primary care providers: a systematic review. J Am Med Inform Assoc 2015 Jan;22(1):236-42.

42. Mozaffar H, Williams R, Cresswell K, Morrison Z, Bates DW, Sheikh A. The evolution of the market for commercial computerized physician order entry and computerized decision support systems for prescribing. J Am Med Inform Assoc 2016 Mar;23(2):349-55.

43. McCoy AB, Wright A, Sittig DF. Cross-vendor evaluation of key user-defined clinical decision support capabilities: a scenario-based assessment of certified electronic health records with guidelines for future development. J Am Med Inform Assoc 2015 Sep; 22(5): 1081-8.

44. Wright A, Hickman TT, McEvoy D, Aaron S, Hussain S, Ramoni R, et al. Analysis of clinical decision support system malfunctions: a case series and survey. J Am Med Inform Assoc 2016 Nov;23(6):1068-76.

45. Chen JH, Goldstein MK, Asch SM, Altman RB. Usability of an automated recommender system for clinical order entry. AMIA Annu Symp Proc 2016;2016:27-8

46. Simpao AF, Tan JM, Lingappan AM, Gálvez JA, Morgan SE, Krall MA. A systematic review of near real-time and point-of-care clinical decision support in anesthesia information management systems. J Clin Monit Comput 2016 Aug 16. [Epub ahead of print]

47. Dunn Lopez K, Gephart SM, Raszewski R, Sousa V, Shehorn LE, Abraham J. Integrative review of clinical decision support for registered nurses in acute care settings. J Am Med Inform Assoc 2016 Jun 21

48. O'Connor PJ, Sperl-Hillen JM, Fazio CJ, Averbeck BM, Rank BH, Margolis KL. Outpatient diabetes clinical decision support: current status and future directions. Diabet Med 2016 Jun;33(6):734-41.

49. Lee EK, Liu Y, Pietz FH. A Compartmental model for Zika virus with dynamic human and vector populations. AMIA Annu Symp Proc 2016;2016:743-52.

50. Ma S, Galatzer-Levy IR, Wang X, Fenyö D, Shalev AY. A first step towards a clinical decision support system for post-traumatic stress disorders. AMIA Annu Symp Proc 2016;2016:837-43.

51. Zaher NA, Buckingham CD. Moderating the influence of current intention to improve suicide risk prediction. AMIA Annu Symp Proc 2016;2016:1274-82.

52. Panny AS, Huser V. Text processing of clinical research protocols and informed consents to facilitate tracking of research procedures. AMIA Annu Symp Proc 2016;2016:1546.

53. Cuggia M, Campillo-GImenez B, Bouzille G, Besana P, Jouini W, Dufour JC, et al. Automatic selection of clinical trials based on a semantic web approach. Stud Health Technol Inform 2015;216:564-8.

54. Blum D, Raj SX, Oberholzer R. Computer-based clinical decision support systems and patient-reported outcomes: a systematic review. Patient 2015 Oct;8(5):397-409.

55. Gallagher J, Rivera R, Chiu A, Tanvir Roushan T, Tanimul Ahsan GM, Weng C, et al. The use of a mHealth decision tree support program for epinephrine autoinjector (eai) administration training of adolescents. AMIA Annu Symp Proc 2016;2016:1399.

56. Thompson JS, Matlock DD, Mcllvennan CK, Jenkins AR, Allen LA. Development of a Decision Aid for Patients with Advanced Heart Failure Considering a Destination Therapy Left Ventricular Assist Device. JACC Heart Fail 2015 Dec;3(12):965-76.

57. Sawka AM, Straus S, Rodin G, Tsang RW, Brierley JD, Rotstein L, et al. Exploring the relationship between patients' information preference style and knowledge acquisition process in a computerized patient decision aid randomized controlled trial. BMC Med Inform Decis Mak 2015;15:48.

58. Center for Medicare and Medicaid Services. Beneficiary engagement and incentive models: direct decision support model. Fact sheet 2016-12-08. https://www.cms.gov/Newsroom/ MediaReleaseDatabase/Fact-sheets/2016-Factsheets-items/2016-12-08.html\#_ftn7. Accessed 10 March 2017.

59. Brown CL, Mulcaster HL, Triffitt KL. A systematic review of the types and causes of prescribing errors generated from using computerized provider order entry systems in primary and secondary care. J Am Med Inform Assoc 2016 Aug 30.

60. Topaz M, Seger DL, Lai K, Wickner PG, Goss F, Dhopeshwarkar N, et al. High override rate for opioid drug-allergy interaction alerts: current trends and recommendations for future. Stud Health Technol Inform 2015;216:242-6.

61. Jafapour B, Abidi SR, Ahmad AM, Abidi SSR. INITIATE: An Intelligent Adaptive Alert Environment. Stud Health Technol Inform 2015;216:285-9.

62. Schreiber R, Gregoire JA, Shaha JE, Shaha SH. Think time: a novel approach to analysis of clinicians' behavior after reduction of drug-drug interaction alerts. Int J Med Inform 2017;97:59-67.

63. Zhou L, Collins S, Morgan SJ, Zafar N, Gesner EJ, Fehrenbach M, et al. A decade of experience in creating and maintaining data elements for structured clinical documentation in EHRs. AMIA Annu Symp Proc 2016;2016:1293-1302.

64. Zhang Y, Jiang M, Wang J. Semantic role labeling of clinical text: comparing syntactic parsers and features. AMIA Annu Symp Proc 2016;2016:1283-92.

65. Rahman P, Lai AM, Hebert CL. Parsing complex microbiology data for secondary use. AMIA Annu Symp Proc 2016;2016:1560.

66. Weitkamp A, Madani S, Rosenbloom TS. Use of an Interface Terminology for Evaluating Terminology Coverage for a Clinical Decision Support System. AMIA Annu Symp Proc 2016;2016:1636.

67. Collins SA, Gesner E, Morgan S, Mar P, Maviglia S, Colburn D, et al. A practical approach to governance and optimization of structured data elements. Stud Health Technol Inform 2015;216:7-11.
68. Hamilton W, Walter FM, Rubin G, Neal RD. Improving early diagnosis of symptomatic cancer. Nat Rev Clin Oncol 2016;13(12):740-9.

69. Besenczi R, Tóth J, Hajdu A. A review on automatic analysis techniques for color fundus photographs. Comput Struct Biotechnol J 2016;14:371-84.

70. Lieng M, Kuhn B, Rehm G, Guo E, Delplanque JP, Anderson N, et al. An automatic, multi-algorithm approach to classify ventilator waveforms. AMIA Annu Symp Proc 2016;2016:83-4.

71. Herasevich V, Dziadzko MA, Aakre CA. Evaluation of clinical score calculator programmability within the electronic health record. AMIA Annu Symp Proc 2016;2016:1426.

72. Huser V, Sastry C, Breymaier M, Idriss A, Cimino JJ. Standardizing data exchange for clinical research protocols and case report forms: An assessment of the suitability of the Clinical Data Interchange Standards Consortium (CDISC) Operational Data Model (ODM). J Biomed Inform 2015 Oct; 57: 88-99.

73. Marco-Ruiz L, Bellika JG. Semantic interoperability in clinical decision support systems: a systematic review. Stud Health Technol Inform 2015;216:958.

74. Jenders RA. Utility of the Fast Healthcare Interoperability Resources (FHIR) standard for representation of non-query data mappings in the Arden Syntax. AMIA Annu Symp Proc 2016;2016:1449.

75. McCallie Jr DP, Mandel J, Strasberg HR, Narus SP, Shekleton K, Marshall P. Beyond SMART: Remote decision support with CDS Hooks. AMIA Annu Symp Proc 2016;2016:2121.

76. Koller W, de Bruin JS, Rappelsberger A, Adlassnig KP. Advances In infection surveillance and clinical decision support with fuzzy sets and fuzzy logic. Stud Health Technol Inform 2015;216:295-9.

77. Jenders RA. Utility of Arden Syntax for representation of fuzzy logic in clinical quality measures. Stud Health Technol Inform 2015;216:1096.

78. Jenders RA, Adlassnig KP, Fehre K, Haug P. Evolution of the Arden Syntax: key technical issues from the standards development organization perspective. Artif Intell Med. 2016 Aug 11.

79. Heale BS, Overby CL, Del Fiol G, Rubinstein WS, Maglott DR, Nelson TH, et al. Integrating genomic resources with electronic health records using the HL7 Infobutton standard. Appl Clin Inform 2016 Aug 31;7(3):817-31.

80. Middleton B, Sittig DF, Wright A. Clinical decision support: a 25 year retrospective and a 25 year vision. Yearb Med Inform 2016; Suppl 1:S103-S116.

81. Peleg M, Tu S. Decision support, knowledge representation and management in medicine. Methods Inf Med 2006;45 Suppl 1:S72-80.

Correspondence to:

Robert A. Jenders, MD, MS, FACP, FACMI

Center for Biomedical Informatics

Charles Drew University

University of California, Los Angeles

1731 E $120^{\text {th }}$ Street

Los Angeles, CA 90059 USA

E-mail: jenders@ucla.edu 